Slavica

bruxellensia

\section{Slavica bruxellensia}

Revue polyphonique de littérature, culture et histoire

slaves

$6 \mid 2010$

Linguistique russe

\title{
L'état physique dans tous ses états
}

Parallèles franco-russes

\section{Irina Kokoškina}

\section{(2) OpenEdition}

\section{Journals}

\section{Édition électronique}

URL : http://journals.openedition.org/slavica/346

DOI : 10.4000/slavica.346

ISSN : 2034-6395

Éditeur

Université libre de Bruxelles - ULB

\section{Édition imprimée}

Pagination : $31-40$

ISSN : 2031-7654

\section{Référence électronique}

Irina Kokoškina, «L'état physique dans tous ses états », Slavica bruxellensia [En ligne], 6 | 2010, mis en ligne le 15 juin 2010, consulté le 19 avril 2019. URL : http://journals.openedition.org/slavica/346 ; DOI : 10.4000/slavica.346

Ce document a été généré automatiquement le 19 avril 2019.

\section{(c)}

Les contenus de Slavica bruxellensia sont mis à disposition selon les termes de la Licence Creative Commons Attribution - Pas d'Utilisation Commerciale - Pas de Modification 3.0 France. 


\title{
L'état physique dans tous ses états
}

\section{Parallèles franco-russes}

\author{
Irina Kokoškina
}

1 Cet article est consacré aux prédicats adjectivaux qui expriment l'état physique relatif à un sujet humain: les <états de forme>, les <états d'ébriété>, les <états de fatigule>, les $<$ états de veille ou de sommeil>, les <états de vie ou de mort>, etc.

2 Les langues confrontées sont le russe et le français. Nous avons volontairement choisi de confronter deux langues qui appartiennent à deux familles différentes, l'une slave et l'autre romane. Nous essaierons de démontrer que les prédicats d'états physiques peuvent être décrits, dans ces deux langues de structure différente, au moyen de critères d'analyses communs. Cette approche permet de révéler un grand nombre de parallèles ${ }^{1}$ entre le russe et le français.

\section{Introduction}

3 La notion d'état a suscité une abondante bibliographie. De nombreuses recherches visent à définir globalement les états par une série de traits sémantiques ${ }^{2}$, d'autres traitent de telle ou telle classe sémantique spécifique ${ }^{3}$. Cependant, la plupart des travaux mentionnés portent sur les prédicats verbaux, alors que les prédicats adjectivaux sont relégués au second plan. Par ailleurs, à la différence des autres états, les états physiques n'ont pas encore fait l'objet d'une étude particulière et ils sont rarement mentionnés dans les travaux qui font désormais référence. Ces deux raisons expliquent le pourquoi de cette étude.

4 Afin de décrire les états physiques ${ }^{4}$, nous allons utiliser la grille d'analyse des états en général que nous avons proposé dans d'autres ouvrages ${ }^{5}$.

5 Nous allons commencer par définir les termes de base de cette étude, à savoir « état » et « prédicat ». 


\section{1. État}

6 Le terme "état » pourrait faire l'objet d'une étude à part entière. Les chercheurs qui travaillent dans le domaine du russe et du français lui attachent des valeurs différentes, souvent contradictoires, en fonction du cadre théorique dans lequel ils se placent. Nous avons dès lors choisi de partir d'une définition formelle des états. L'article porte ainsi sur les unités linguistiques qui entrent dans le schéma suivant:

$7 \quad$ No by ̌r Pred en russe

No être Pred en français

8 «Pred » renvoyant au prédicat et « N0 » représentant le sujet. Il s'agira d'adjectifs simples et composés ${ }^{6}$.

\subsection{Prédicat}

9 La base théorique du présent article est constituée par les recherches de Zellig Sabbettai Harris ${ }^{7}$. Conformément à celles-ci, l'unité minimale d'analyse est la phrase simple, à savoir le prédicat accompagné des arguments qui en dépendent.

Considérons deux exemples suivants, l'un russe, l'autre français :

Oleg byl mërtv, nikakikh somnenij. / Oleg était mort, il n'y avait aucun doute possible.

(MarininaA., Ukradennyjson ${ }^{8}$ [Le Rêve volé]).

Dans ces phrases, mërtv et mort sont considérés comme prédicats, alors qu'Oleg constitue un argument, en l'occurrence, argument-sujet.

\section{Analyse des états physiques}

11 L'article est centré sur cinq critères de description des $\mathrm{PEP}^{9}$ : la classe sémantique, le temps, l'aspect, l'intensité et l'évaluation. Comme nous l'avons annoncé dans notre introduction, nous allons cibler notre analyse sur les éléments d'ordre sémantique et distributionnel qui sont communs au russe et au français. Pour les besoins d'analyse, les exemples russes seront systématiquement accompagnés de leur traduction en français.

\subsection{Classe sémantique}

Le critère de la classe sémantique est primordial pour notre analyse dans la mesure où, d'après nos calculs, l'ensemble des états regroupe environ deux cent classes différentes. Il est donc impossible d'employer le terme "état " sans préciser la classe sémantique à laquelle appartient le prédicat.

Par exemple :

-prédicats de <couleur> : oranževyj (orange), zelënyj (vert), etc.- prédicats de <forme> : ovaL'nyj (ovale), kruglyj (rond), etc.

- <aspect physique > : khudoj (maigre), tolstyj (gros), etc.

- <états d'ordre et de désordre > : v Modif. ${ }^{10}$ Porjadke (en Modif. ordre), v Modif. Besporjadke (en Modif. désordre, ordonné, rangé), etc.

- <états de propreté> : čistyj (propre), grjaznyj (sale), etc.

- <états de conscience> : $v$ kome (dans le coma), bez soznanija (sans connaissance), etc. 
on peut y distinguer les classes suivantes :

- <états de vie ou de mort> živ (en vie), mërtv (mort)

- <états de fatigue $>^{11}$ ustalyj (fatigué), zamučennyj (exténué), etc.

- <états de forme ${ }^{12}>\mathrm{v}$ Modif. forme (dans une forme Modif.), ne $v$ forme (pas en forme), $v$ tonuse (tonique), etc.

- <états de sommeil> vo sne (endormi), v polusne (à moitié endormi), vob'jatijakh Morfeja (dans les bras de Morphée), etc.

- <états d'ébriété> p'janyj (ivre), v sostojanii Modif. op'janenija (dans un état d'ébriété), etc.

4 Nous venons de citer quelques lexèmes qui ont servi de matériau pour cette étude. L'établissement des classes sémantiques est à plus d'un titre la condition sine qua non de l'analyse. Comme on peut s'en rendre compte dans l'exemple cité ci-dessus, les PEP constituent un ensemble très hétérogène. Établir les classes nous a permis de réunir les prédicats homogènes sémantiquement et possédant les mêmes propriétés distributionnelles.

\subsection{Temps}

russe et en français, la plupart des états, et notamment des états physiques, n'existent pas en dehors du temps. L'information temporelle est exprimée avant tout à l'aide des verbes supports, à savoir byŤ (ou ses équivalents, par exemple nakhodiŤsja) en russe et être (ou ses équivalents, par exemple se trouver, etc.) en français.

Par exemple :

Dans l'exemple cité ci-dessus, l'information temporelle est contenue dans les verbes nakhodiŤsja et se trouver. Ces verbes permettent de déterminer sur l'axe temporel la place de l'état exprimé par les prédicats $v$ otmennoj fizičeskoj formei et dans une forme physique optimale. Mais l'information temporelle peut également être apportée par les adverbes (ou les locutions adverbiales). Considérons les adverbes včera, segodnja et leurs analogues français. Par exemple :

Dans ces phrases, la valeur des adverbes déictiques est proche de celle qui est assumée par les verbes nakhodiŤsja et se trouver dans les exemples cités auparavant. Včera et hier, segodnja et aujourd'hui traduisent la localisation temporelle. Cependant, une information supplémentaire est également présente. Le locuteur n'insiste pas uniquement sur le fait que l'état a lieu au moment du discours, il précise également sa place sur l'axe temporel. En d'autres mots, la temporalité est exprimée deux fois dans ces exemples, de deux façons différentes.

Dans les phrases citées ci-dessus, les verbes aussi bien que les adverbes constituent des marqueurs temporels. Ceux-ci sont possibles avec la majorité des prédicats d'états (et notamment avec les prédicats d'états physiques) en russe et en français. Il convient cependant de faire la remarque suivante : certains prédicats traduisent une propriété qui caractérise le sujet tant que celui-ci existe. Dans ce cas, seuls les verbes by̌̌ et être, à valeur atemporelle, sont possibles :

19 Les marqueurs temporels sont interdits dans ce contexte pour des raisons sémantiques. Kruglaja et ronde décrivent ici une caractéristique inaliénable des sujets Zemlja et la Terre. Celle-ci pourrait être appelée définitionnelle, dans la mesure où elle conditionne l'existence du sujet lui-même. De façon logique, la caractéristique définitionnelle n'est 
pas soumise au facteur temporel. Or, la possibilité d'employer un adverbe temporel sousentend que la caractéristique est susceptible de changement. De la sorte, le prédicat et l'adverbe apportent une information contraire, qui est à l'origine du blocage.

20 Le critère du temps nous a permis de séparer deux types de prédicats. Cette dichotomie s'observe dans les deux langues analysées. Les uns expriment les états qui ont lieu dans le temps, c'est le cas de la majorité des PEP. Les autres renvoient à un état des choses invariable. Ci-dessous, nous démontrerons que le facteur du temps conditionne le comportement linguistique d'un prédicat donné face au critère de l'aspect.

\subsection{Aspect}

21 Le terme "aspect » tel qu'il sera utilisé ici renvoie aussi bien à l'aspect au sens strict qu'aux modes d'action. Notre analyse des valeurs aspectuelles des prédicats en français et en russe s'appuie sur les travaux de Gaston Gross ${ }^{16}$. Le terme « aspect interne » désigne les limites temporelles de l'état, alors que le terme " aspect externe » renvoie aux diverses phases de celui-ci. Il s'agira des marqueurs inchoatifs, continuatifs et terminatifs, qui, dans les langues analysées, mettent en valeur le début, la suite ou la fin de l'état, respectivement.

22 Les états sont traditionnellement associés à la durée (notamment chez Zeno Vendler et Tatjana Bulygina). D'après nos observations, ce constat s'avère vrai aussi bien en russe qu'en français. Presque tous les prédicats d'états, et notamment les PEP, appartiennent à l'aspect interne duratif, ils décrivent des situations qui durent dans le temps. Cette propriété sémantique est corroborée par les possibilités combinatoires desdits prédicats. Ils acceptent aisément divers marqueurs de restriction temporelle.

Par exemple :

23 Dans ces exemples, les locutions temporelles ves' den'et pendant toute la journée précisent la durée de l'état physique exprimé par ustavšij et fatigué.

Considérons un autre exemple :

NeskoL'ko dnej on byl meždu žizn'ju i smerŤju, i posle neverno primenënnoj vanny umer ot razryva serdca. / Pendant plusieurs jours, il fut entre la vie et la mort et, après un bain administré à mauvais escient, il mourut d'une crise cardiaque.

(BaL'mont K., Belaja nevesta ${ }^{17}$ [La fiancée blanche])

Ici, il s'agit des prédicats de la classe <états de vie ou de mort> et les marqueurs temporels (neskol'ko dnej et pendant plusieurs jours) fixent les limites temporelles de l'état.

Les exemples démontrent que le critère de durée est applicable à tous les prédicats, et ceci dans les deux langues analysées. Un fait attire cependant notre attention. Lorsqu'il s'agit d'une caractéristique atemporelle, les marqueurs temporels sont exclus. Les prédicats de propriété définitionnelle (dont il a été question ci-dessus) ne passent pas le test de la combinaison avec les adverbes et locutions adverbiales à valeur temporelle :

6 Le blocage est lié au fait que la qualité kruglaja (ronde en français) fait partie de la substance du sujet Zemlja (Terre). La qualité en question ne peut pas être représentée sur l'axe temporel. De la sorte, la valeur de la locution dva goda (pendant deux ans) entre en contradiction avec celle du prédicat.

Ci-dessus nous avons démontré que la combinaison avec les adverbiaux de temps est conditionnée par le fait que le prédicat exprime un état dont la durée est mesurable. Cette affirmation est primordiale pour les deux langues. En effet, ces prédicats sont les seuls à 
accepter les marqueurs d'aspect externe. Compte tenu des limites de cet article, nous nous limiterons à certaines remarques concernant l'emploi de tel ou tel marqueur. La première remarque concerne la relation de dépendance entre l'aspect interne et l'aspect externe. En russe et en français, les prédicats qui n'acceptent pas les adverbes de durée (auxquels on ne peut pas appliquer le terme d'aspect interne) ne permettent pas non plus les marqueurs d'aspect externe.

Par exemple :

Les marqueurs de début (stă̌ [devenir]) et de fin (perestaŤ [cesser de]) sont ici en contradiction, par leur sémantisme, avec la valeur du prédicat (propriété définitionnelle). La deuxième remarque est la suivante. Lorsque le marqueur d'aspect externe est possible, sa nature dépend de la classe sémantique à laquelle appartient le prédicat. Considérons les <états de sommeil>. Pour des raisons de place, nous nous limiterons à deux exemples. Le début de l'état est exprimé au moyen des verbes de mouvement employés métaphoriquement. Par exemple pogruziŤsja (se plonger) :

No ja uže ne smogla ničego otvetit' i pogruzilas' $v$ sladkie

ob'jatija Morfeja. / Mais je n'ai plus rien pu répondre et j'ai plongé dans les bras de Morphée. <états de sommeil>(Doncova D., Čërt iz tabakerki ${ }^{19}$ [Le Diable de la tabatière])

30 À ce niveau, les états physiques peuvent être mis en relation avec les états psychologiques qui, d'après la juste remarque de George Lakoff ${ }^{20}$, sont conceptualisés par l'être humain comme des milieux liquides. Cette remarque, faite par le chercheur à partir des exemples anglais, s'avère également vraie pour le russe et le français. Une analyse détaillée des marqueurs inchoatifs appliqués aux états physiques permet cependant de faire le constat suivant. Le verbe pogruziřsja met l'accent sur un changement d'état lent. En revanche, d'autres verbes, tels que vpaš̌ (tomber) représentent le changement d'état comme quelque chose de brusque, d'inattendu :

Obratno vyletel iz Pitera v noč na 21. Kak zanjal kreslo, tak $i$ vpal v glubokij son. / Il partit de Pétersbourg par avion la nuit du 21. À peine assis sur son siège, il tomba dans un profond sommeil. <états de sommeil>(Lapšin Ju., Dnevniki i pis'ma ${ }^{21}$ [Journaux intimes et lettres])

Dans ce paragraphe, nous ne nous sommes pas fixé comme objectif de décrire de façon détaillée les marqueurs temporels susceptibles de se combiner avec les états physiques, mais de dégager ce qui semble être une constante qui unit le russe et le français. Nous avons surtout souhaité souligner que la majorité des états (y compris les états physiques) se combinent avec les marqueurs aspectuels. Il existe cependant les états dont la durée n'est pas mesurable, notamment les prédicats de propriété définitionnelle (dont il a été question ci-dessus).

\subsection{Intensité}

Pour décrire les propriétés distributionnelles des PEP du point de vue du critère de l'intensité, il nous est indispensable d'introduire deux termes, scalarité et intensité. Le premier sera utilisé au sens que lui rattache R. Rivara ${ }^{22}$. On entend par scalarité la possibilité du prédicat d'exprimer les degrés de sens (au sens large). Le terme "intensité » se situe sur un plan différent. Nous allons l'employer en décrivant les moyens lexicaux dont dispose une langue (en l'occurrence, le russe et le français) pour exprimer les degrés de sens. 

concernant le fait que tous les prédicats d'états ne sont pas scalaires. Divers cas de figure doivent être distingués. D'abord, les marqueurs d'intensité sont exclus si le prédicat exprime intrinsèquement le haut degré d'une qualité ou d'un état.

Par exemple :

41 L'impossibilité d'employer ici les adverbes d'intensité tels que očen' (très) tient à la nature sémantique des prédicats qui, par leur nature, constituent des sortes de superlatifs. Cela explique le caractère peu naturel des exemples cités. Lorsqu'unadverbededegréestcombiné auprédicat, on aboutit à la redondance.

42 Le cas de figure cité ci-dessus n'est pas isolé. Il existe d'autres prédicats non-scalaires. Il s'agit notamment de ceux qui forment des séries ${ }^{24}$, par exemple les états physiques de la classe <états de vie ou de mort> živ - mërtv (en vie - mort) :

Une fois cette distinction posée, nous allons pouvoir exposer les résultats auxquels nous sommes parvenus au terme de notre analyse. Le premier constat qui s'impose est que, en russe et en français, l'ensemble des états peut être divisé en deux groupes, états scalaires et états non-scalaires. Considérons de ce point de vue les <états de fatigue> :

Nous dirons que ces états sont scalaires. Cette propriété sémantique possède un corrélat au niveau distributionnel. Les lexèmes cités acceptent les adverbes de degré :

extrêmement, jouent le rôle de marqueurs d'intensité. Ils expriment ici le haut degré de la qualité exprimée par les prédicats ustavšij (en russe) et fatigué (en français). De façon logique, les prédicats non scalaires ne peuvent pas apparaître dans des contextes similaires. En effet, l'idée même d'intensité, contenue dans le marqueur, serait en contradiction avec le sens de ces prédicats qui, par définition, excluent les degrés de sens. scalaires et non scalaires n'est pas suffisante pour les décrire de façon détaillée. Une analyse plus fine s'impose dans la mesure où chacune des classes possède ses particularités. L'exempleV tot večer ja byl (očen' + črezvyčajno) ustavšij mettaitenvaleurles étatsphysiques (<états de fatigue>) et nous nous sommes limitée à quelques marqueurs. Si nous considérons les <étatsdesommeil>, ici aussi les marqueurs sont spécifiques :

l'état physique. Le locuteur a recours à la métaphore, glubokij relevant, dans son sens premier, du domaine des adjectifs paramétriques.

prédicats d'états physiques. Ainsi, dans le cas des <états de fatigue>, on relève les constructions avec les verbes pada ̌ ot (tomber de), umiră̌ ot (mourir de), etc. Par exemple : fatigue) expriment le haut degré de l'état de fatigue.

Nous allons maintenant de revenir à l'observation que nous avons faite ci-dessus L'impossibilité de gradation de ces prédicats est d'ordre sémantique, mais elle est également liée à la nature du référent. Les prédicats mentionnés ne peuvent pas se combiner avec les adverbes de degré, comme il en ressort de l'exemple suivant :

Les prédicats qui composent des séries décrivent des états contraires. Si l'un estutilisé, l'autreest automatiquement exclu. 
Enterminantcesous-chapitreconsacré à l'intensité, ilconvientdenoterque les fondements épistémiques de l'intensité sont divers. Chaque classe sémantique doit êtreconsidéréeséparément, cequiconfirmel'extrême importance du paramètre d'intensité dans l'analyse des états physiques (et des états en général).

\section{5. Évaluation}

$$
\text { problèmes d'acceptabilité. En revanche, la combinaison avec čuvstvovař sebja et se sentir }
$$
sont considérés par les locuteurs comme étant douteuse ${ }^{26}$.

\section{Conclusion}

$$
\text { connaissance, fait l'objet d'une étude particulière. Il s'agit d'un travail inédit qui met en }
$$
parallèle les deux langues mentionnées.

53 À l'étape actuelle de notre recherche, il nous est impossible de répondre à toutes les questions qui concernent les états physiques. Nous avons dès lors ciblé notre analyse sur cinq critères. Ceux-ci ont permis de mettre en évidence le fait que les deux langues 
révèlent plusieurs points de rapprochement. Ainsi, le critère de la classe sémantique a permis d'éclairer la structuration sémantique des PEP. Celle-ci est à grands traits similaire en russe et en français, tant au niveau du nombre des classes qu'au niveau de la nature des éléments qui les composent. Le critère du temps a révélé que la grande majorité des PEP en russe et en français exprime un état qui occupe un intervalle limité sur l'axe temporel. L'exception à ce niveau sont les prédicats de propriétés définitionnelles. Le paramètre du temps est indissolublement lié à celui de l'aspect. La plupart des PEP en russe et en français décrivent des situations qui durent dans le temps et qui, de ce fait, se combinent avec les marqueurs de restriction temporelle. Les prédicats de propriétés définitionnelles constituent la seule exception à ce niveau, et ceci dans les deux langues. L'analyse des PEP en termes d'intensité a permis de les diviser en deux ensembles, à savoir états scalaires et états non-scalaires. Seuls les premiers acceptent les adverbes de degré et pas les seconds. Au-delà de ce constat général, une analyse plus fine s'impose pour décrire en détail le comportement de chaque classe sémantique prise isolément. Enfin, notre dernier critère c'était l'évaluation. En russe et en français, la plupart des PEP traduisent un état qui peut faire l'objet d'une appréciation. Cependant, il en existe d'autres, statistiquement minoritaires, qui renvoient à un état qui ne peut pas être évalué.

Dans cet article, nous n'avons fait qu'esquisser les principales lignes d'analyse et illustrer certains phénomènes. Il est évident que l'étude détaillée des PEP en russe et en français doit faire appel à d'autres critères, tels que la cause, la fréquence, les propriétés morphologiques, pour n'en citer que certains. Nous comptons approfondir notre analyse et en présenter les résultats dans une publication ultérieure.

\section{NOTES}

1. D'après nos observations, les divergences entre les prédicats d'états physiques en russe et en français ne concernent que certains faits précis. Il ne s'agit nullement de les masquer. Nous leur réservons une publication ultérieure.

2. Ces travaux se basent souvent sur la classification de Zeno Vendler donnée in : Vendler Z., Linguistics in Philosophy, Cornell University Press, Ithaca-New York, 1967, 203 p. Ainsi, Bulygina T., «K postroeniju tipologii predikatov v russkom jazyke » (Vers la typologie des prédicats en russe), in: Semantičeskie tipy predikatov (Types sémantiques de prédicats; sous la direction de Selivërstova O.), Nauka, Moscou, 1982, pp.7-85 et Padučeva E., Semantičeskie issledovanija. Semantika vremeni $i$ vida $v$ russkom jazyke (Recherches sémantiques. La sémantique du temps et de l'aspect en russe), Škola «Jazyki russkoj kuL'tury ", Moscou, 1996, 464 p. définissent les états en russe par les traits suivants: stativité, durativité et absence de contrôle. D’autres chercheurs (Miller J., "Stative verbs in Russian» (Les Verbes statiques en russe), in : "Foundations of LANGUAGE ", t.6, D. Riedel Publishing Company, Dordrecht, 1970, pp. 488-504. pour le russe et Riegel M., L'Adjectif attribut, Presses universitaires de France, Paris, 1985, 222 p. pour le français) prennent comme point de départ les critères établis par George Lakoff : les verbes statifs ne peuvent pas être mis à l'impératif, ils n'acceptent pas les adverbes de manière, etc. Pour le compte rendu détaillé des études sur le russe, nous renvoyons à Akmalova F., Semantičeskaja $i$ 
formaL'no-strukturnaja reprezentacija kategorii sostojanie na materiale anglijskogo, nemeckogo i russkogo jazykov (Représentation sémantique, formelle et structurelle de la catégorie d'état en anglais, en allemand et en russe), thèse de doctorat, Iževsk, 2005, 235 p. pour le russe et à Kokoškina I., Typologie des prédicats d'états, Thèse de doctorat, Université Paris 13, 2004, 392 p. pour le français.

3. Parmi tous les états, ce sont avant tout les états psychologiques (ou encore « sentiments ", ou "affects», selon l'auteur) qui ont fait l'objet d'études, notamment pour le français: Van de Velde D., « Les verbes dits "psychologiques" revus à la lumière des noms correspondants ", in : «REVUe De LinguistiQue RomAne», t.59, G. Straka, Strasbourg, pp.67-98; Van de Velde D., "Adjectifs d'états, adjectifs de qualités", in : Fonctions syntaxiques et rôles sémantiques (Sous la direction de Amiot D. et alii), Presses Universitaires d'Arras, Artois, pp. 151-160. ; Leeman D., «Tentative de caractérisation d'un complément circonstanciel : Dans mon affolement, je lâchai mon panier de cerises", in: "LINX ", t. 12,Centre de recherches linguistiques de Paris 10, Nanterre, 1985, pp.97-145; Leeman D., «Hurler de rage, rayonner de bonheur: remarques sur une construction en "de" », in : «LANGUe FRANÇAISE », t. 91, Armand Colin, Paris, 1991, pp. 80-101; Leeman D., « Pourquoi peut-on dire Max est en colère mais non *Max est en peur ? », in : « LANGUE FRANÇAISE », t. 105, Armand Colin, Paris, 1995, pp. 55-69. Pour le russe : Cejtlin St., « Sintaksičeskie modeli so značeniem psikhologičeskogo sostojanija i ikh sinonimika » (Modèles syntaxiques pour exprimer l'état psychologique et leur synonymes), in : Sintaksis i stilistika (Syntaxe et stylistique ; sous la direction de Zolotova G.), Moscou, 1976, pp. 161-181 ; Vol'f E., « EmocionaLnye sostojanija i ikh predstavlenie v jazyke» (Les états émotionaux et leur représentation dans la langue), in : Logičeskij analiz jazyka. Problemy intensionaL’nykh i pragmatičeskikh kontekstov (Analyse logique de la langue. Problèmes des contextes intentionnels et pragmatiques; sous la direction de Arutjunova N. \& Rjabceva N.), Nauka, Moscou, 1989, pp.55-74 ; Zaliznjak A., Issledovanija po semantike predikatov vnutrennego sostojanija (Recherches sur le sémantisme des prédicats d'états interne), O. Sagner, Munich, 1992, 201 p.; Kurlova I., «K izučeniju glagol’nykh sposobov vyrazenija emocionaL'nykh sostojanij » (Éléments pour l'étude des verbes d'état psychologique), in: «SLOVAR'. GRAMMATIKA. TEXT. »,Rossijskaja akademija nauk, otdelenie literatury i jazyka, Moscou, 1996, $472 \mathrm{p}$.

4. Nous utiliserons plus tard l'abréviation «PEP ».

5. La grille d'analyse comprend quatorze critères, à savoir la nature des arguments, la classe sémantique, le temps, l'aspect, la fréquence, la cause, les propriétés morphologiques, la reprise anaphorique, l'interrogation, la non-agentivité, l'évaluation, l'intensité, les séries temporelles et les classifieurs.

6. Certains états physiques sont exprimés par des participes. Nous les citerons également.

7. Harris Z. S., Notes du cours de syntaxe, Éd. du Seuil, Paris, 1976, 236 p.

8. Eksmo-Press, Moscou, 2000, 230 p.

9. Pour l'ensemble des critères nous renvoyons aux sources citées à la note 4 .

10. «Modif.» indique ici le modifieur, par exemple l'adjectif qui permet au locuteur de caractériser l'état physique du sujet humain. Par exemple « olympique » dans dans une forme olympique.

11. Ces prédicats peuvent également renvoyer à un état mental.

12. Dans certains contextes, les prédicats mentionnés désignent la forme psychologique de l'individu. C'est le contexte linguistique ou extralinguistique qui permet de lever l'ambiguïté.

13. http://www.vibirai.ru/lipetsk/article/.../408586.html, page consultée le 2 mars 2010.

14. Proposer des exemples russes et les mettre en parallèle avec les exemples français correspondants (vérifiés auprès des locuteurs natifs) constitue une démarche volontaire de notre part et elle est motivée par le souci de donner au lecteur qui ne sait pas le russe le moyen de comparer valablement. Au niveau méthodologique, nous considérons, suite à E. Laporte («Exemples attestés et exemples construits dans la pratique du lexique-grammaire», in: « 
MÉmoires De la Société De linguistiQue DE PARIS », t.16, Peeters, Louvain/Paris/Dudley, 2008, pp. 11-32) que les méthodes "expérimentale» et "observationnelle» sont complémentaires. Nous travaillons dès lors avec les exemples attestés aussi bien qu'avec les exemples construits.

15. À noter cependant: Ran'še Zemlja byla ploskoj (Autrefois la Terre était ronde), où la phrase exprime la doctrine officielle qui existait auparavant.

16. Gross G., « Prédicats nominaux et compatibilité aspectuelle », in : «LANGAGES », t. 121, Armand Colin, Paris, 1996, pp. 54-73.

17. http://fb2lib.net.ru/read_online/101517. Page consultée le 15 juin 2010.

18. À noter cependant : Avec Galilée, la Terre est devenue ronde.

19. http://www.aldebaran.ru/det/donc/donc18. Page consultée le 19 juin 2010.

20. http://metaphor.narod.ru/lacoff_6.htm. Page consultée le 15 juin 2010.

21. http://militera.lib.ru/db/lapshin_um/index.html. Page consultée le 15 juin 2010.

22. Rivara R., "Adjectifs et structures sémantiques scalaires", in: " L'INFORMATION GRAMmATiCALE », t. 58, Peeters, Paris, 1993, pp. 40-46.

23. http://az.lib_ru/o/odoewskij_w_f/text_0060.shtml. Page consultée le 15 juin 2010.

24. Voir Leduc-Adine J.-P., "Polysémie des adjectifs de couleur », in : «CAHIERS DE LEXICOLOGIE ", t. 37, Honoré Champion, Paris, 1980, p. 73

25. « Trésor de la Langue Française », v. 13, Gallimard, Paris, 1985-1994, p. 305.

26. La phrase On čuvstvoval sebja mërtvym ne nous semble pas totalement impossible. Elle pourrait être prononcée dans un contexte particulier, métaphorique, où le locuteur porte un jugement sur son état.

\section{AUTEUR}

\section{IRINA KOKOŠKINA}

Maître de conférences à l'Université Paris IV 